6 - ORIGINAL ARTICLE

WOUND HEALING

\title{
Low intensity ultrasound therapy induces angiogenesis and persistent inflammation in the chronic phase of the healing process of third degree burn wounds experimentally induced in diabetic and non-diabetic rats ${ }^{1}$
}

\author{
Marcelo Silva Fantinati', Diego Eterno Oliveira Mendonça', Adriana Márcia Monteiro Fantinati" ${ }^{\mathrm{III}}$, Bruno Flamarion dos \\ Santos $^{\text {IV }}$, Juliana Cristina Oliveira Reis ${ }^{\text {Iv }}$, Cristina Lopes Afonso ${ }^{\text {, }}$ Marina Clare Vinaud ${ }^{\text {VI, Ruy de Souza Lino Júnior }}$ \\ DOI: http://dx.doi.org/10.1590/S0102-865020160070000006 \\ IPhD, Full Professor, Superior School of Physical Education and Physical Therapy of Goias State (ESEFFEGO), Universidade Estadual de Goiás \\ (UEG), Goias-GO, Brazil. Intellectual, scientific, conception and design of the study;manuscript writing.

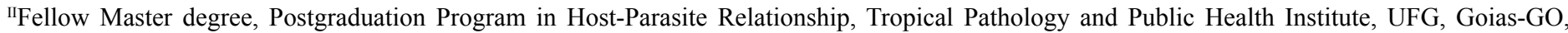 \\ Brazil. Acquisition of data. \\ IIIMaster, Assistant Professor, ESEFFEGO, UEG, Goias-GO, Brazil. Acquisition of data. \\ ${ }^{\mathrm{IV}}$ Graduate student, Physical Therapy, ESEFFEGO, UEG, Goias-GO, Brazil. Acquisition of data. \\ ${ }^{v}$ Specialist, Assistant Professor, ESEFFEGO, UEG, Goias-GO, Brazil. Conception and design of the study. \\ VIPhD, Associate Professor, Tropical Pathology and Public Health Institute, Universidade Federal de Goiás (UFG), Goias-GO, Brazil. Analysis and \\ interpretation of data, manuscript writing. \\ VIIPhD, Associate Professor, Tropical Pathology and Public Health Institute, UFG, Goias-GO, Brazil Scientific and intellectual content of the study, \\ analysis and interpretation of data, statistical analysis, manuscript preparation.
}

\begin{abstract}
PURPOSE: To evaluate the effects of low intensity ultrasound on the healing process of third degree burn wounds in experimentally induced diabetic Wistar rats.

METHODS: One hundred rats were divided into: control group; non-diabetic treated group; diabetic control group; diabetic treated group. The therapy was performed with a $3 \mathrm{MHz}$ ultrasound application, pulsed emission at $100 \mathrm{~Hz}$ frequency, modulated at $20 \%$ with a dosage of $0.5 \mathrm{~W} / \mathrm{cm}^{2}$ during three minutes throughout 30 days. The surgical debridement of the wound was performed once at day 2 . The wounds were morphometrically, macroscopically and microscopically evaluated at 3, 7, 14, 21 and 30 days.

RESULTS: The wound contraction and collagen quantification were higher in all treated groups. Macroscopically, necrosis was higher in the diabetic control group. Granulation tissue was higher in treated groups during the proliferative and remodeling phase. Microscopically, there were greater mononuclear inflammatory infiltration, angiogenesis and fibroblast quantification in treated groups during the proliferative and remodeling phases.
\end{abstract}

CONCLUSIONS: therapeutic ultrasound is beneficial in the inflammatory and proliferative phases of the healing process because it controlled the necrotic tissue, increased the granulation tissue and wound contraction. However in the remodeling phase it is not beneficial because of the continued angiogenesis and a mononuclear inflammatory infiltration.

Key words: /ultrasonography. Burns. Wound Healing. Diabetes Mellitus, Experimental. Rats. 


\section{Introduction}

The evolution of the healing process involves a series of phenomena which represent attempts in reestablishing the anatomical structure and function of the normal tissue ${ }^{1}$. During these phenomena several systemic and local factors are involved and the unbalance or the absence of elements, caused by the injury type and/or by the reduction of blood supply may compromise the final healing result ${ }^{2}$.

Burn wounds, especially third degree ones, associated with the metabolic state of diabetes mellitus (DM) are factors that impair the normal evolution of tissue repair due to the complexity of the injury with expressive alteration of a number of structures and due to the compromise in the blood supply to the affected region $^{3}$. The importance of experimental studies of burn wounds have been highlighted by Schmauss et al. ${ }^{4}$ in a review evaluating different models used and the importance of the investigation of pathologic findings.

It is estimated that $9.3 \%$ of the North American population presents with $\mathrm{DM}^{5}$. Also DM is the $7^{\text {th }}$ leading cause of death in the USA $^{5}$. While Europe has a prevalence of $8.5 \%$ of DM patients ${ }^{6}$. In Central and South America it was estimated that DM is present in 26.4 million people ${ }^{7}$. It is estimated that Brazil will rise from the $8^{\text {th }}$ position in the ranking with $5.6 \%$ of DM prevalence to the $6^{\text {th }}$ position with $11.3 \%$ in $2030^{8}$.

Developing countries are the ones which present the highest incidences of burn wounds ranging from 1.3/100.000 inhabitants in low income countries ${ }^{9}$. In Brazil, the Health Ministry reports that burn wounds are in the $4^{\text {th }}$ place as a cause of death due to injury and it is estimated that 1.000 .000 people in Brazil suffer some kind of burn wound each year ${ }^{10}$.

According to Bertoldi et al. ${ }^{8}$ there is no epidemiological relation between burn wounds and DM. However it is known that $2 / 3$ of the diabetic population suffer burn wounds and that they present delayed healing processes.

Several different treatments are proposed to help the tissue repair such as polymers, phytotherapic extracts, mineral compounds, physical resources such as low level laser therapy, therapeutic ultrasound (US), light emitting diode and hyperbaric chambers $^{3}$. Other authors report that under normal conditions therapeutic US favors wound contraction, angiogenesis and fibroblasts migration to the injury bed ${ }^{1,11}$. Nevertheless, other studies describe that the therapeutic US accelerates the proliferative phase of wound repair through the stimulating effect on the inflammatory mediators ${ }^{12}$.

Low intensity US is frequently used in the treatment of injuries of soft tissues ${ }^{13}$. It may also be applied in the wound healing process especially because it is a non-invasive nonthermal technique with low cost and efficacy at a cellular level including angiogenesis, leukocyte adhesion, growth factor and collagen production, as well as increases in macrophage responsiveness, fibrinolysis and nitric oxide levels ${ }^{14}$. Therefore the aim of this study was to analyze the healing process of burn wounds in experimentally induced diabetic rats after low intensity US treatment.

\section{Methods}

This study was approved by the Ethics Committee in Animal Use (CEUA/PRPPG-UFG) protocol number 007/12. The ethical principles for animal experimentation of the Sociedade Brasileira de Ciência em Animais de laboratório (SBCAL) were followed ${ }^{15}$.

Three animals were housed in each cage. They received water and autoclaved commercial ration ad libitum and the bed changes were performed twice a week. An adaptation of the animals in the proposed environment was performed so as to prioritize their well being.

\section{Experimental Type I Diabetes Mellitus (DM) induc- tion}

For the DM induction an intraperitoneal injection of streptozotocine dissolved in citrate buffer $0.1 \mathrm{M}, \mathrm{pH}=4.5,(40 \mathrm{mg} /$ $\mathrm{kg}$ ), was performed ${ }^{16}$. The evaluation of the vascular commitment was performed through measure of the thickness of the splenic white pulp central arteriole ${ }^{17}$. The animals were considered diabetic when they developed glucose levels higher than $200 \mathrm{mg} /$ $\mathrm{dl}$ and when they developed thickness of the splenic white pulp central arteriole. The glucose levels were determined by glucose meter device $\left(\right.$ Biocheck $\left.^{\circledR}\right)$ from a drop of blood from the tail of the animal at 24 and 72 hours after the DM induction procedure and at the euthanasia day.

After the DM confirmation a histopathologic analysis of the central arteriole from the white pulp of the spleen was performed aiming the detection of vascular alterations induced by this disease. The spleen of the animals $(n=3)$ was removed at 2, 15 and 30 days after the DM induction and the tissues were processed for paraffin inclusion and two slides of 4 micrometer width each were stained with hematoxilin \& eosin (HE) and picrosirius. The thickness of the splenic white pulp central arteriole was determined through microscopic analysis of HE stained slides by using the Image J software (NIH) (data not shown). 


\section{Experimental groups}

One hundred 60-75 days old female Wistar rats weighing 200-250g were used. The animals were divided into four groups of 25 animals each: control group (CG); diabetic group (DG); nondiabetic treated group (NDTG) and diabetic treated group (DTG). A pilot study was conducted as to evaluate the vascular alterations induced by DM, therefore other three animals were used in the evaluation of the splenic white pulp central arteriole as described above. The total number of animals used was 103 .

Five animals from each group were euthanized at 3, 7, 14, 21 and 30 days after the induction (DAI) of the third degree burn wound for the macroscopic, microscopic and morphometric analysis of the wound. Therefore each experimental group presented a subgroup division according to Figure 1.

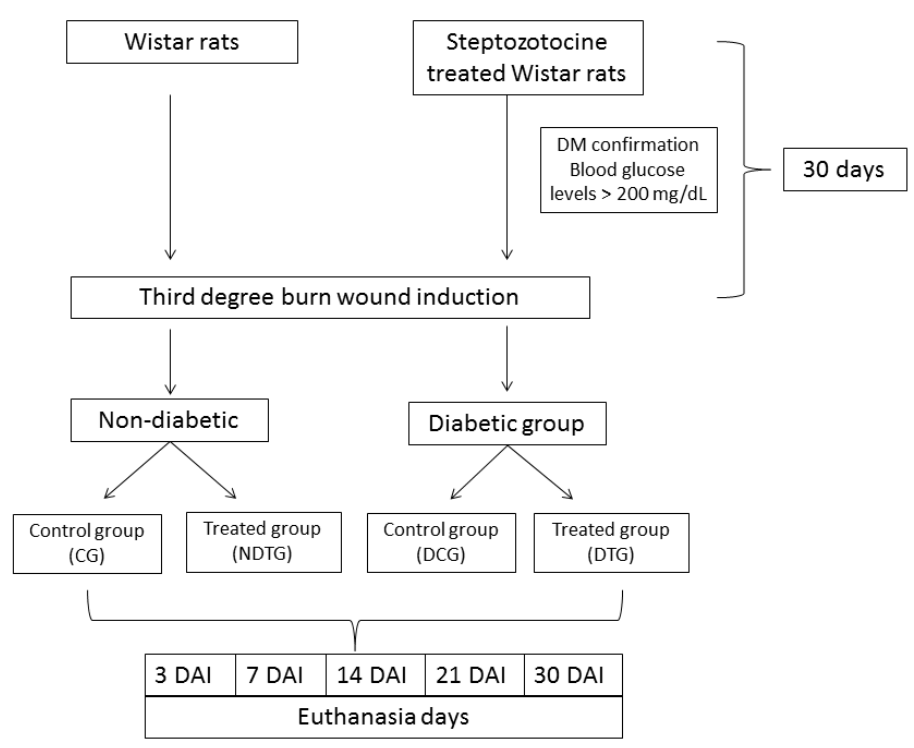

FIGURE 1 - Schematic representation of groups distribution. DM: diabetes mellitus. CG: control group; NDTG: non diabetic treated group; DCG: diabetic treated group; DTG: diabetic treated group; DAI: days after the injury induction.

The euthanasia of these animals was performed by peritoneal injection of $1 \mathrm{ml} / \mathrm{g}$ of Ketamine $10 \%$ and Xilazine $2 \%$. All analyses were performed by a researcher blinded to the groups.

\section{Third degree burn wound induction}

The burn wound induction occurred after 30 days of the DM induction, when the glucose levels and the two fold increase in the thickness of the splenic white pulp central arteriole confirmed the experimental diabetes and the vascular commitment. After local preparation of the dorsal skin, shaving and antisepsis, the animals were anesthetized with $0.01 \mathrm{ml} / \mathrm{g}$ of Ketamine $10 \%$ and Xilazine $2 \%$ via intraperitoneal. The third degree thermal injury was induced as described previously by Moraes et al. ${ }^{18}$. Briefly, each animal was placed inside a PVC (polyvinyl chloride) plastic cylinder with a $2 \times 2 \mathrm{~cm}$ opening and sealed extremities, the uncovered area was immersed in water at $95^{\circ} \mathrm{C}$ for 14 seconds. A pilot study performed previously showed that the immersion of the rat dorsal area for 14 seconds caused the burn injury of the epidermis, dermis and hypodermis confirming a third degree burn wound with necrosis and disarrangement of collagen fibers (data not shown).

During the treatment period the animals (control and treated groups) received daily occlusive and sterilized bandages. Initially the cleaning of the wound with physiologic solution was performed followed by a topic application of silver sulfadiazine ointment. All animals received an occlusive bandage. The injured animals were supervised by a veterinarian physician during the animal hygiene when aggressiveness, posture and respond to handling were observed.

According to the literature the treatment of burn wounds is based on the early wound debridement and closure ${ }^{4}$. Therefore, at the second day after the injury induction the surgical debridement of the wounds occurred in all animals. The surgical debridement was performed only once with a scalpel as to remove all the necrotic tissue. No chemical debridement was used ${ }^{2}$.

\section{Therapeutic US treatment}

The US equipment, model SONOMED V 4150, used in this study was developed by Carci Indústria e Comércio de Aparelhos Cirúrgicos e Ortopédicos Ltda (Americanópolis - São Paulo - SP - Brasil). The therapeutic US treatment began at day 0 immediately after the burn wound induction. The animals were gently manipulated and stabilized by the researchers and it occurred daily with direct perpendicular application of the ultrasound soundhead on the wound area which was covered with coupling hydrogel. The sonographic application was performed in a circulatory manner with an operating frequency of $3 \mathrm{MHz}$, pulsed emission at a frequency of $100 \mathrm{~Hz}$ modulated at $20 \%$ with dosage of $0.5 \mathrm{~W} / \mathrm{cm}^{2}$. The probe remained on the wound area for 3 minutes. The pressure was enough to allow contact of the soundhead with the hydrogel and the wound as to promote the conductance of the sonographic application ${ }^{13,14}$. 


\section{Macroscopic and wound contraction analysis}

At the established experimental days the phases of the inflammatory process were macroscopically evaluated, i.e., inflammation, proliferation and maturation. The following parameters were evaluated: necrosis and granulation tissue. The validation of the methodology was performed through the macroscopic evaluation of the injured skin which showed changed in coloration (pale/milky) which was confirmed in the microscopic analysis that showed necrotic tissue and disarrangement of collagen fibers (data not shown).

For the wound contraction analysis the wounds were photographed after $3,7,14,21$ and 30 days with a digital camera coupled to a tripod and kept at a fixed distance of $20 \mathrm{~cm}$ of the injury. Afterwards the images were analyzed through the Image $J$ software (NIH) version 1.3.1.

The wound area was contoured through the Image J software by a researcher who did not know which treatment was used. Afterwards the wound contraction degree was calculated through the following mathematic equation: $\mathrm{CG}=\left(\right.$ area $\mathrm{D}^{0}$ - area $\left.\mathrm{D}^{\prime}\right) \mathrm{x} 100 /$ area $\mathrm{D}^{0}$, where $\mathrm{CG}$ is contraction degree, $\mathrm{D}^{0}$ is the initial area at day $0, D^{\prime}$ is the area at the experimental day $(3,7,14,21$ or 30). Therefore was possible to determine the differences in injured area throughout the experimental days.

\section{Microscopic analysis}

The injured tissue was removed through biopsy, processed for paraffin inclusion, sectioned at 4 micrometers width and stained with hematoxilin \& eosin (HE). The samples of cutaneous lesion collected including the skin adjacent to the injury edges and the scar tissue in its depth were analyzed.

The general pathological processes were evaluated in the dermis and injury edge. The analysis of this lesions was qualitative where the parameters were: absent (A) (attributed score 0 ); discrete (D) in which 1 to $25 \%$ of the analyzed slide presented the alteration (attributed score 1); moderate (M) in which 26 to
$50 \%$ of the analyzed slide presented the alteration (attributed score 2) and accentuated (Ac) in which more than $51 \%$ of the analyzed slide presented the alteration (attributed score 3 ). Afterwards the attributed score was converted into mean \pm standard deviation ${ }^{18}$.

The pathological processes analyzed in the dermis were necrosis, fibrin, hemorrhage, polymorphonuclear (PMN) infiltration, mononuclear (MN) infiltration, hyperemia, angiogenesis and fibroblasts.

For the morphometric analysis of the connective tissue the skin fragments were fixed and embedded in paraffin, stained with picro-sirius and counter staining with hematoxilin at 14, 21 and 30 days after the injury induction. The slides were analyzed through a circular polarized light microscope (Zeiss $\left.{ }^{\circledR}\right)$ with the 10x objective and the morphometry was performed using the Image $\mathrm{J}$ software. 20 fields were quantified which approximately corresponded to the whole area of the slide.

\section{Statistical analysis}

All variables were tested as to the normal distribution and homogenous variation. When the distribution was considered normal and with homogenous variation the t-Student test was performed. In the cases in which there was not a normal distribution the Mann-Whitney test was used. The differences were considered significant when $\mathrm{p}<0.05$. The statistical analysis was performed using the Sigma Stat software version 3.5.

\section{Results}

\section{Wound contraction and macroscopic analysis}

The morphometric analysis of the diabetic and nondiabetic animals showed that the groups that received the therapeutic US treatment had a significantly higher wound contraction than the non-treated groups $(\mathrm{p}<0.05)$ (Table 1$)$.

TABLE 1 - Percentage of third degree wound contraction in diabetic and non-diabetic Wistar rats treated with therapeutic US.

\begin{tabular}{|c|c|c|c|c|c|c|c|c|c|c|}
\hline & \multicolumn{2}{|c|}{3 DAI } & \multicolumn{2}{|c|}{7 DAI } & \multicolumn{2}{|c|}{14 DAI } & \multicolumn{2}{|c|}{$21 \mathrm{DAI}$} & \multicolumn{2}{|c|}{30 DAI } \\
\hline & Mean (\%) & SD & Mean (\%) & SD & Mean (\%) & SD & Mean (\%) & SD & Mean \%) & SD \\
\hline $\mathbf{C G}$ & 9.2 & 0.1 & 14.5 & 0.6 & 63.7 & 0.6 & 87.4 & 1.9 & 94.9 & 2.5 \\
\hline \multirow[t]{2}{*}{ NDTG } & 10.5 & 0.8 & 28.7 & 0.7 & 74.2 & 0.8 & 92.3 & 1.0 & 99.6 & 0.4 \\
\hline & \multicolumn{2}{|c|}{$\mathrm{p}=0.036^{*}$} & \multicolumn{2}{|c|}{$\mathrm{p}=0.001 *$} & \multicolumn{2}{|c|}{$\mathrm{p}=0.001^{*}$} & \multicolumn{2}{|c|}{$\mathrm{p}=0.003^{*}$} & \multicolumn{2}{|c|}{$\mathrm{p}=0.005^{*}$} \\
\hline DG & 1.0 & 0.2 & 10.6 & 0.5 & 54.1 & 1.2 & 81.0 & 1.0 & 88.2 & 2.7 \\
\hline \multirow[t]{2}{*}{ DTG } & 2.0 & 0.7 & 42.4 & 3.1 & 72.6 & 5.1 & 91.7 & 0.5 & 99.4 & 0.6 \\
\hline & \multicolumn{2}{|c|}{$p=0.626$} & \multicolumn{2}{|c|}{$\mathrm{p}=0.001 *$} & \multicolumn{2}{|c|}{$\mathrm{p}=0.001 *$} & \multicolumn{2}{|c|}{$\mathrm{p}=0.001 *$} & \multicolumn{2}{|c|}{$\mathrm{p}=0.001 *$} \\
\hline
\end{tabular}

Legend: DAI = days after the injury induction; SD = standard deviation; CG: non-diabetic control group; NDTG: non-diabetic treated group; DG: diabetic group; DTG: diabetic treated group, ${ }^{*} \mathrm{p}<0.05$, statistic test: Student's t test, comparison between treated and control groups. 
In the evolution of the healing process of the third degree burn wounds (Figure 2, Table 2) the diabetic control group had significantly higher intensity of necrosis at 3 DAI, in the inflammatory phase. In the proliferative phase this significant difference in the necrosis intensity was maintained at 7 DAI accompanied by significant increase in the granulation tissue in the non-diabetic treated group. At 14 DAI there was a significant increase in the granulation tissue in the non-diabetic treated group. In the remodeling phase, at 21 DAI, a "Y" shaped healing was observed.

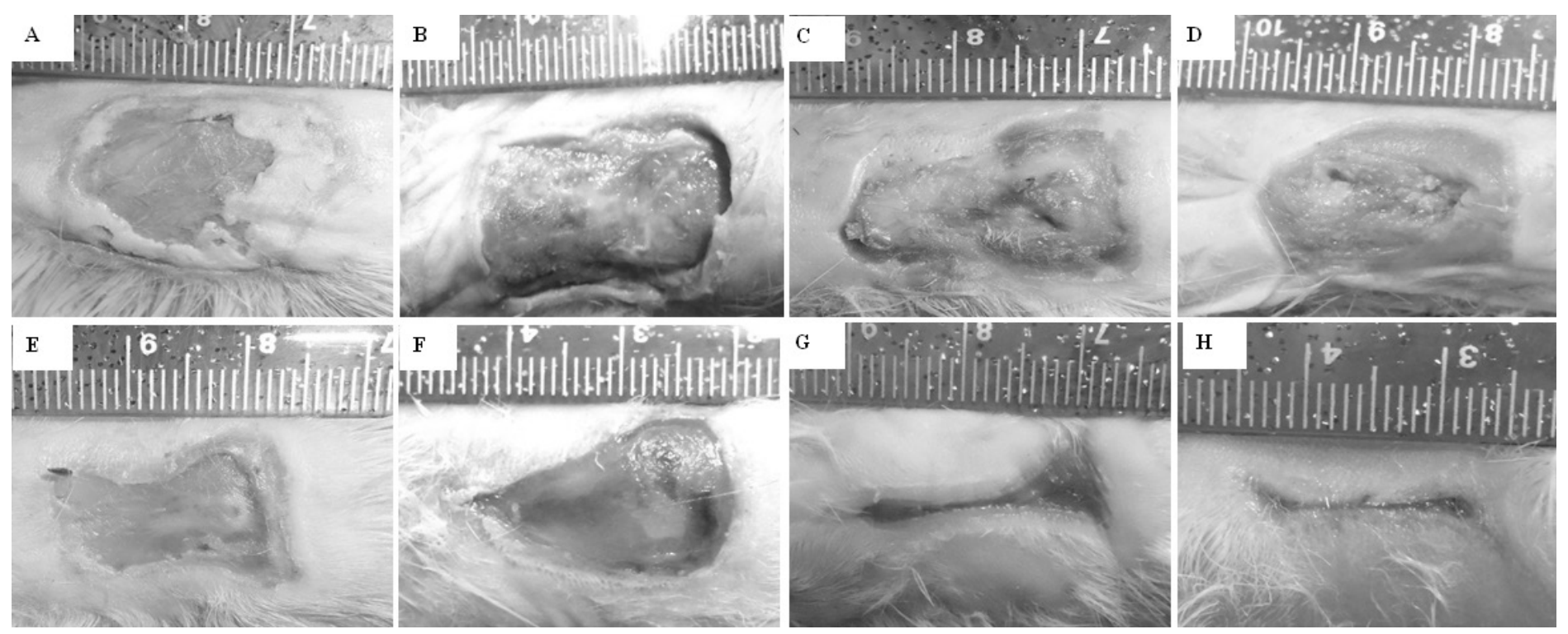

FIGURE 2 - Macroscopic photographs of the wound contraction of third degree burn wound in diabetic and non-diabetic Wistar rats. At 3 days after injury induction, (A) diabetic control group; (B) diabetic treated group. At 7 days, (C) control group; (D) non-diabetic treated group. At 14 days after injury induction, (E) control group; (F) non-diabetic treated group. At 21 days; (G) control group; (H) non-diabetic treated group.

TABLE 2 - Macroscopic qualitative analysis of the general pathologic processes in third degree burning wounds in experimentally induced diabetic Wistar rats after therapeutic Ultrasound treatment. Results in mean \pm standard deviation.

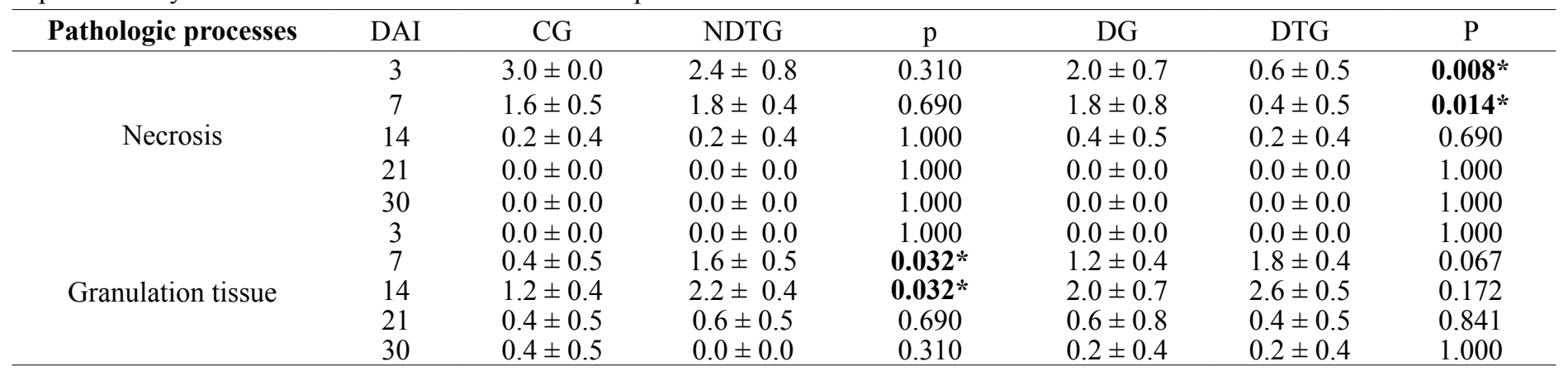

Legend: DAI = days after the injury induction; CG: non-diabetic control group; NDTG: non-diabetic treated group; DG: diabetic group; DTG: diabetic treated group. Statistic test: Mann-Whitney, comparison between treated and control groups.

\section{Microscopic analysis}

The general pathologic processes analysis was performed comparing the treated and non-treated groups (Figure 3, Table 3). In the inflammatory phase (3 DAI) the therapeutic US treatment induced a significant increase in angiogenesis and in fibroblasts in the non-diabetic group. In the proliferative phase (7 and 14 DAI) the treatment induced a significant increase in the mononuclear $(\mathrm{MN})$ inflammatory infiltration, angiogenesis and fibroblasts in the non-diabetic group. In the diabetic group the treatment induced a significant increase in fibroblasts at 14 DAI. In the remodeling phase (21 and $30 \mathrm{DAI}$ ) the treatment induced a significant increase in the $\mathrm{MN}$ inflammatory infiltration and angiogenesis in the diabetic group and an increase in the $\mathrm{MN}$ inflammatory infiltration in the non-diabetic group. Still at $21 \mathrm{DAI}$ there was a significant increase in angiogenesis and fibroblasts in the non-diabetic treated group. 


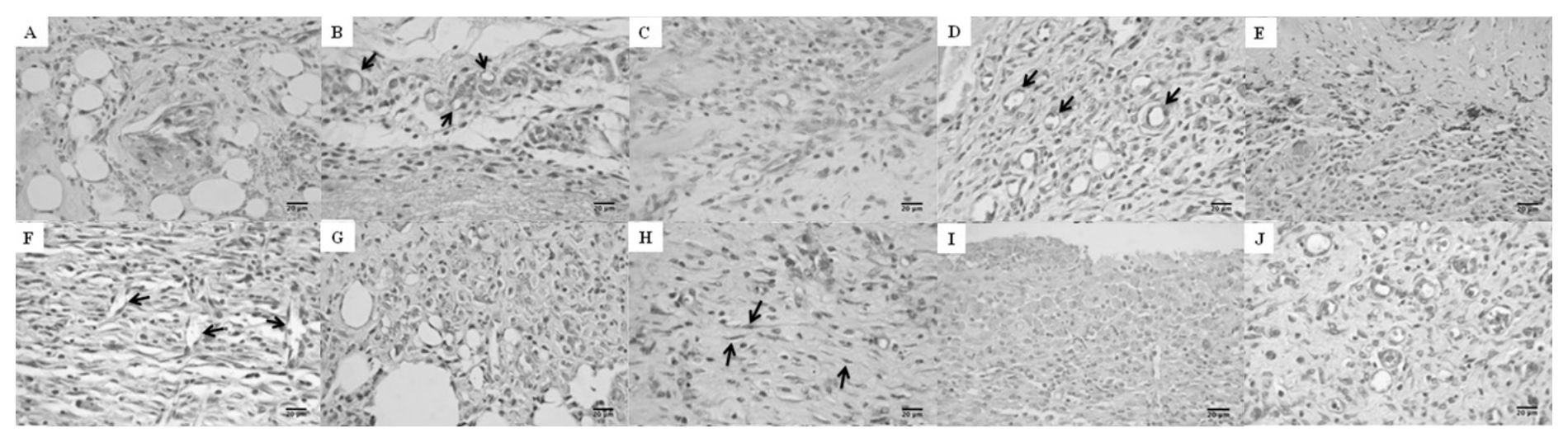

FIGURE 3 - Photomicrograph of the third degree burn wound in Wistar rats. In non-diabetic animals at 3 days, (A) control group with lower angiogenesis (arrow) and fibroblasts when compared to (B) non-diabetic treated group. At 7 days, (C) control group with lower angiogenesis (arrow), fibroblasts and mononuclear cells infiltration when compared to $(\mathbf{D})$ non-diabetic treated group. At 21 days, $(\mathbf{E})$ control group with lower angiogenesis (arrow), fibroblasts and mononuclear cells infiltration when compared to (F) non-diabetic treated group. In diabetic animals, at 14 days, $(\mathbf{G})$ diabetic group with lower fibroblasts (arrow) when compared to $(\mathbf{H})$ diabetic treated group. At 21 days, (I) diabetic group with lower mononuclear cells infiltration when compared to $(\mathbf{J})$ diabetic treated group.

TABLE 3 - Microscopic qualitative analysis of the general pathologic processes in third degree burning wounds in experimentally induced diabetic Wistar rats after therapeutic Ultrasound treatment. Results in mean \pm standard deviation.

\begin{tabular}{|c|c|c|c|c|c|c|c|}
\hline Pathologic processes & DAI & CG & NDTG & $\mathrm{P}$ & $\mathrm{DG}$ & DTG & $\mathrm{p}$ \\
\hline \multirow{5}{*}{ Necrosis } & 3 & $3.0 \pm 0.0$ & $3.0 \pm 0.0$ & 1.000 & $3.0 \pm 0.0$ & $3.0 \pm 0.0$ & 1.000 \\
\hline & 7 & $2.0 \pm 0.0$ & $2.0 \pm 0.0$ & 1.000 & $2.0 \pm 0.0$ & $2.0 \pm 0.0$ & 1.000 \\
\hline & 14 & $1.8 \pm 0.4$ & $1.8 \pm 0.4$ & 1.000 & $1.8 \pm 0.4$ & $1.8 \pm 0.4$ & 1.000 \\
\hline & 21 & $0.2 \pm 0.4$ & $0.2 \pm 0.4$ & 1.000 & $0.6 \pm 0.5$ & $0.6 \pm 0.5$ & 1.000 \\
\hline & 30 & $0.0 \pm 0.0$ & $0.0 \pm 0.0$ & 1.000 & $0.4 \pm 0.5$ & $0.4 \pm 0.5$ & 1.000 \\
\hline \multirow{5}{*}{ PMN infiltration } & 3 & $2.0 \pm 0.0$ & $2.0 \pm 0.0$ & 1.000 & $2.2 \pm 0.4$ & $2.2 \pm 0.4$ & 1.000 \\
\hline & 7 & $1.4 \pm 0.5$ & $1.6 \pm 0.5$ & 0.690 & $1.2 \pm 0.4$ & $1.2 \pm 0.4$ & 1.000 \\
\hline & 14 & $0.6 \pm 0.8$ & $1.2 \pm 0.4$ & 0.222 & $0.6 \pm 0.5$ & $1.0 \pm 0.0$ & 0.310 \\
\hline & 21 & $0.4 \pm 0.5$ & $1.2 \pm 0.4$ & 0.095 & $0.0 \pm 0.0$ & $0.2 \pm 0.4$ & 0.690 \\
\hline & 30 & $0.0 \pm 0.0$ & $0.0 \pm 0.0$ & 1.000 & $0.0 \pm 0.0$ & $0.0 \pm 0.0$ & 1.000 \\
\hline \multirow{5}{*}{ MN infiltration } & 3 & $0.2 \pm 0.4$ & $0.4 \pm 0.5$ & 0.690 & $0.6 \pm 0.5$ & $0.6 \pm 0.5$ & 1.000 \\
\hline & 7 & $0.6 \pm 0.5$ & $2.0 \pm 0.0$ & $0.008 *$ & $0.6 \pm 0.5$ & $0.6 \pm 0.5$ & 1.000 \\
\hline & 14 & $1.2 \pm 0.4$ & $3.0 \pm 0.0$ & $0.008 *$ & $1.8 \pm 0.8$ & $2.6 \pm 0.5$ & 0.111 \\
\hline & 21 & $1.2 \pm 0.4$ & $2.2 \pm 0.4$ & $0.032 *$ & $2.0 \pm 0.0$ & $3.0 \pm 0.0$ & $0.008 *$ \\
\hline & 30 & $0.6 \pm 0.5$ & $1.8 \pm 0.4$ & $0.016 *$ & $1.0 \pm 0.0$ & $2.0 \pm 0.0$ & 0.008* \\
\hline \multirow{5}{*}{ Angiogenesis } & 3 & $0.2 \pm 0.4$ & $1.4 \pm 0.5$ & $0.016 *$ & $0.2 \pm 0.4$ & $0.2 \pm 0.4$ & 1.000 \\
\hline & 7 & $0.6 \pm 0.8$ & $2.6 \pm 0.5$ & $0.016 *$ & $2.4 \pm 0.8$ & $2.4 \pm 0.8$ & 1.000 \\
\hline & 14 & $1.2 \pm 0.4$ & $2.8 \pm 0.4$ & $<0.001 *$ & $2.4 \pm 0.8$ & $3.0 \pm 0.0$ & 0.310 \\
\hline & 21 & $1.4 \pm 0.5$ & $2.8 \pm 0.4$ & $0.002 *$ & $1.4 \pm 0.5$ & $3.0 \pm 0.0$ & $0.008 *$ \\
\hline & 30 & $1.6 \pm 0.8$ & $2.8 \pm 0.4$ & $0.028 *$ & $1.6 \pm 0.8$ & $3.0 \pm 0.0$ & $0.032 *$ \\
\hline \multirow{5}{*}{ Fibroblast } & 3 & $0.0 \pm 0.0$ & $1.6 \pm 0.5$ & $0.008 *$ & $0.0 \pm 0.0$ & $0.6 \pm 0.5$ & 0.151 \\
\hline & 7 & $0.0 \pm 0.0$ & $2.8 \pm 0.4$ & $0.008 *$ & $1.4 \pm 0.5$ & $1.6 \pm 0.5$ & 0.310 \\
\hline & 14 & $1.2 \pm 0.4$ & $3.0 \pm 0.0$ & $0.008 *$ & $1.4 \pm 0.5$ & $3.0 \pm 0.0$ & $0.008 *$ \\
\hline & 21 & $1.6 \pm 0.8$ & $3.0 \pm 0.0$ & $0.032 *$ & $2.4 \pm 0.5$ & $3.0 \pm 0.0$ & 0.151 \\
\hline & 30 & $2.2 \pm 0.8$ & $3.0 \pm 0.0$ & 0.151 & $2.4 \pm 0.5$ & $3.0 \pm 0.0$ & 0.151 \\
\hline
\end{tabular}

Legend: DAI = days after the injury induction; CG: non-diabetic control group; NDTG: non-diabetic treated group; DG: diabetic group; DTG: diabetic treated group, PMN: polimorphonuclear cells; MN: mononuclear cells. Statistic test: Mann-Whitney, comparison between treated and control groups.

The collagen quantification was significantly higher in the treated groups when compared to the non-treated ones (Table 4). 
TABLE 4 - Percentage of collagen deposition in experimentally induced diabetic Wistar rats with third degree burn wounds. Results expressed in median (minimum - maximum).

\begin{tabular}{|c|c|c|c|c|c|c|}
\hline & 14 DAÍ & & 21 DAÍ & & $30 \mathrm{DAI}$ & \\
\hline $\mathrm{CG}$ & $\begin{array}{c}10.16 \\
(9.08-13.65) \\
12.72 \\
(7.38-19.92)\end{array}$ & $\mathrm{p}<0.001$ & $\begin{array}{c}10.27 \\
(9.08-12.55) \\
20.59 \\
(19.24-22.30)\end{array}$ & $p<0.001$ & $\begin{array}{c}10.23 \\
(9.08-11.88) \\
20.96 \\
(19.91-21.79)\end{array}$ & $\mathrm{p}<0.001$ \\
\hline DG & $\begin{array}{c}10.17 \\
(9.17-12.49) \\
16.18 \\
(9.95-19.13)\end{array}$ & $p<0.001$ & $\begin{array}{c}10.11 \\
(9.06-12.48) \\
14.15 \\
(9.99-22.64)\end{array}$ & $p<0.001$ & $\begin{array}{c}10.15 \\
(9.57-13.94) \\
19.54 \\
(9.85-21.55)\end{array}$ & $\mathrm{p}<0.001$ \\
\hline
\end{tabular}

Legend: DAI = days after the injury induction; CG: non-diabetic control group; NDTG: non-diabetic treated group; DG: diabetic group; DTG: diabetic treated group, statistical test: Mann-Whitney, comparison between control and treated groups.

\section{Discussion}

This study shows an innovative approach for the treatment of third degree burn wounds in an experimental model with rats. The morphometric, macroscopic and microscopic features of the healing process of diabetic Wistar rats treated with therapeutic US were analyzed. The results show that the therapeutic US treatment is beneficial in the inflammatory and proliferative phases of the healing process due to the control of the necrotic tissue, induction of a significant increase in the granulation tissue and in the wound contraction. However in the remodeling phase it may not be beneficial because it stimulates continuous angiogenesis and mononuclear inflammatory infiltration.

The beneficial effect of the wound debridement has been described previously in a different wound mode ${ }^{19}$. Also debridement of the wound is important to remove the necrotic tissue and the bacterial proliferation in the dead tissue ${ }^{19}$. Silver sulfadiazine cream has been used in the wound dressings to prevent infection and maintain a moist environment at the injured site and has also reduced pain in patients ${ }^{20}$. While some studies suggest that silver sulfadiazine may delay epithelialization it is still considered the standard procedure in many burn studies and centers ${ }^{21}$.

Ward et al..$^{22}$ with a different scheme of topical therapeutic ultrasound treatment, $1 \mathrm{MHz}$, continuous ultrasound with $5 \mathrm{~cm}$ soundhead for a period of $10 \mathrm{~min}$ at an intensity of $1.0 \mathrm{w} / \mathrm{cm}^{2}$ every other day, concluded that patients did not show improvement in range of motion nor in pain. Unfortunately the anatomopathologic aspects of these treated lesions were not observed. However Sari et al. ${ }^{23}$ reported an improvement in range of motion in burn patients treated with $1 \mathrm{MHz}$, intensity of $1 \mathrm{~W} / \mathrm{cm}^{2}$ applied with a $5 \mathrm{~cm}$ applicator for 5 minutes, five sessions per week during 3 weeks. Therefore the frequency of the therapeutic ultrasound treatment application influenced in its result. In our study the therapeutic ultrasound was applied daily leading to favorable results in the wound healing. Cambier and Vanderstraeten ${ }^{24}$ used the same therapeutic US frequency in experimental third degree burn wounds as in our study, however the protocol of burn wound induction and the subsequent treatment of the third degree burn wound were not the same as the ones we used. The former authors did not find differences in the contraction and in the histological analysis (data not shown). It is possible that these differences occurred because we used a different protocol for the third degree burn wound treatment which was composed of surgical debridement followed by daily dressings of occlusive bandages embedded in silver sulfadiazine.

The effects of therapeutic US in humans ${ }^{19}$ are similar to those found in this study. This supports the use of this experimental model for the proposed therapeutic methodology. Also Mahran ${ }^{14}$ showed that the preferred power density for the therapeutic US treatment is $0.5 \mathrm{~W} / \mathrm{cm}^{2}$, which induced a higher contraction of wounds, similar to the findings in our study. Altomare et al. ${ }^{11}$ showed that the wound contraction was higher in the treated group only during the proliferative phase of healing in a study with a simple surgical injury with no alteration in the wound contraction in comparison with a group with surgical injury by peripheral tissue ischemia. These data partly differ from our study in which all treated group had a significant difference in wound contraction both in diabetic and non-diabetic groups.

Regarding the macroscopic analysis, the necrosis found in the inflammatory and proliferative phases was significantly higher in the control group when compared to the diabetic treated group. These results differ from other studies because in our study there was the debridement of the injury at the first day after the injury induction. This technique was chosen as to improve and accelerate the healing process through the removal of necrotic 
tissue as to diminish the bacterial contamination and therefore improve the action and effect of the longitudinal US emission. Another relevant factor was the use of a humid occlusive bandage because this procedure insured the hydration of the wound which minimized the crust formation ${ }^{12,14}$. It was possible to observe in the therapeutic US treated groups significant differences in the granulation tissue during the inflammatory and proliferative phases in the non-diabetic group.

Mesquita et l $^{25}$ during the 14 days of analysis of lowintensity therapeutic ultrasound in the healing process of third degree burn wounds in rats reported no significant influence of the treatment on angiogenesis, inflammatory infiltration and granulation tissue when comparing treated and non-treated burned groups. Differing from our findings in which the therapeutic ultrasound treatment induced increase in the mononuclear inflammatory infiltration, angiogenesis and collagen formation up to 14 days after the injury induction. In the following experimental days at 21 and 30 days after the burn induction, which are corresponding to the remodeling phase of the healing process, the low-intensity therapeutic ultrasound was not beneficial due to the continued angiogenesis and persistence of the inflammatory infiltration. These differences in the initial phase of the healing process may have occurred due to the fact that those authors did not perform the debridement of the burn wound and did not used occlusive bandages embedded in silver sulfadiazine which are protocols recommended for the treatment of burn wounds.

In the microscopic analysis the main significant differences found were the presence of mononuclear cells especially during the regenerative phase of the healing process; angiogenesis, fibroblasts and collagen increased in both treated groups when compared to their respective control ones. These results are similar to the ones described by other authors who used different parameters, analyzed tissue and experimental models ${ }^{26}$. According to the literature the non thermal (acoustic) effect of the therapeutic US in the emitting region stimulates the biological properties of the injured tissue especially during the inflammatory phase which favors cell migration, as well as cytokine and growth factor production, which induce the formation of new blood vessels, fibroblasts and consequently a new collagen matrix ${ }^{1}$.

This study shows that the collagen deposition was significantly higher in the proliferative and remodeling phases in the treated groups when compared to their respective control ones. Other non-diabetic experimental models performed with rabbits and horses have shown similar results in skin injuries ${ }^{26}$.

During the proliferative phase, the therapeutic US treatment promotes an increase in the number of fibroblast, which results in greater collagen deposit. This probably occurs due to an increase in the cellular membrane permeability ${ }^{27}$. The therapeutic US promotes the reorientation of the collagen fibers leading to a greater elasticity with little loss of the tensile strength. The mechanical properties of the scar are related to the amount of collagen and its rearrangement or alignment within the wound bed ${ }^{19}$. Demir et al..$^{28}$ in a study with non-diabetic rats also showed that the therapeutic US treatment associated with wound debridement and daily care is beneficial during the inflammatory, proliferative and remodeling phases of the wound healing.

In this study the central arteriole in the white pulp of the diabetic rat's spleen was thickened, which is similar to what is observe din humans with diabetic foot ulcers ${ }^{17}$ and in Wistar rats with induced diabetic nephropathy, which present a progressive alteration in all the renal arterioles walls ${ }^{29}$.

This is one of the first studies to describe the use of therapeutic US to improve the healing process in burn wounds in an experimental model of diabetic rats. There are several articles in the literature that use the rat model ${ }^{4,18,27,28}$ in spite of the fact that rats have a subcutaneous panniculus carnosus muscle which contributes to the repair via contraction and collagen formation ${ }^{4,28}$. The increase in wound contraction is beneficial to the healing process as it approximates the wound margins and helps close the injury. The increase in collagen formation in this model is also beneficial to wound closure and healing and did not produce hypertophic scar or keloid ${ }^{30}$.

\section{Conclusions}

Therapeutic ultrasound helped control necrosis and accelerated wound closure by contraction. However, I also led to a significant increase in angiogenesis and inflammation in the proliferative phase of healing which might contribute to the chronicity of wounds.

\section{References}

1. Park NJ, Allen L, Driver VR. Updating on understanding and managing chronic wound. Dermatol Ther. 2013 MayJun;26(3):236-56. doi: 10.1111/dth.12052.

2. Baum CL, Arpey CJ. Normal cutaneous wound healing: clinical correlation with cellular and molecular events. Dermatol Surg. 2005 Jun;31(6):674-86. PMID: 15996419

3. Rosique RG, Rosique MJ, Farina Junior JA. Curbing inflammation in skin wound healing: a review. Int J Inflam. 2015;2015:316235. doi: $10.1155 / 2015 / 316235$.

4. Schmauss D, Rezaeian F, Finck T, Machens HG, Wettstein R, Harder Y. Treatment of secondary burn wound progression in contact burns-a systematic review of experimental approaches. J Burn Care Res. 2015 May-Jun;36(3):e176-89. doi: 10.1097/ BCR.0000000000000131. 
5. Adebayo O, Willis GC. The changing face of diabetes in America. Emerg Med Cin North Am. 2014 May;32:319-27. doi: 10.1016/j. emc.2013.12.004.

6. Schaaf L, Hummel M. Environemtal factors in the pathogeneis of diabetes mellitus. MMW Fortschr Med. 2014 Jan 20; 156(1):58-60. PMID: 24930312.

7. Mudaliar U, Kim WC, Kirk K, Rouse C, Narauan KM, Ali M. Are recommended standards for diabetes care met in Central and South America? A systematic review. Diabetes Res Clin Pract. 2013 Jun;100(3):306-29. doi: 10.1016/j.diabres.2013.01.010.

8. Bertoldi AD, Kanavos P, França GV, Carraro A, Tejada CA, Hallal PC, Ferrario A, Schmidt MI. Epidemiology, management, complications and costs associated with type 2 diabetes in Brazil: a comprehensive literature review. Global Health. 2013 Dec 3;9:62. doi: 10.1186/1744-8603-9-62.

9. Peck MD. Epidemiology of burns throughout the world: Part I: distribution and risk factors. Burns. 2011 Nov;37(7):1087-100. doi: 10.1016/j.burns.2011.06.005.

10. Iqbal T, Saaiq M, Ali Z. Epidemiolgoy and outcome of burns: early experience at the country's first national burns centre. Burns. 2013 Mar;39(2):358-62. doi: 10.1016/j.burns.2012.07.011.

11. Altomare M, Nascimento AP, Souza BR, Amadeu TP, Costa AMA. Ultrasound accelerates healing of normal wounds but not of ischemic ones. Wound Rep Regen. 2009 N0v-Dec;17(6):825-31. doi: 10.1111/j.1524-475X.2009.00542.x.

12. Wei FY, Leung KS, Li G, Qin J, Chow SK, Huang S, Sun MH, Qin L, Cheung WH. Low intensity pulsed ultrasound enhanced mesenchymal stem cell recruitment through stromal derived factor-1 signaling in fracture healing. PLoS One. 2014 Sep 2;9(9):e106722. doi: 10.1371/journal.pone.0106722.

13. Miller DL, Smith NB, Bailey MR, Czarnota GJ, Hynynen K, Makin IR. Bioeffects Committee of the American Institute of Ultrasound in Medicine. Overview of therapeutic ultrasound applications and safety considerations. J Ultrasound Med. 2012 Apr;31(4):623-34. PMID: 22441920

14. Mahran HG. Influence of contact ultrasonic with different power densities on full-thickness wounds healing: an experimental study. Int J Physiother Res. 2014;2(3):567-76.

15. CONCEA (Conselho Nacional de Controle de Experimentação Animal). Para produção, manutenção ou utilização de animais em atividades de ensino ou pesquisa cientifica. 2015. Disponível em: http://www.mct.gov.br/upd_blob/0238/238271.pdf

16. Almeida SM de, Ferreira RI, Bóscolo FN. Influence of irradiation on collagen content during wound healing in diabetic rats. Pesqui Odontol Bras. 2002 Oct-Dec;16(4):293-8. PMID: 12612766.

17. Liew G, Wang JJ. Retinal vascular signs in diabetes and hypertension - review. Arq Bras Endocrinol Metab. 2007 Mar;51(2):352-62. PMID: 17505645.

18. Moraes JM, Mendonça DEO, Moura VBL, Oliveira MAP, Afonso CL, Vinaud MC, Bachion MM, de Souza Lino R Jr. Antiinflammatory effect of low-intensity laser on the healing of thirddegree burn wounds in rats. Lasers Med Sci. 2013 Jul;28(4):116976. doi: 10.1007/s10103-012-1213-1.

19. Amini S, Shojaeefard A, Annabestani Z, Hammami MR, Shaiganmehr Z, Larijani B, Mohseni S, Afshano HR, Rad MA, Mohajeri-Tehrani MR. Low-frequency ultrasound debridement in patients with diabetic foot ulcers and osteomyelitis. Wounds. 2013 Jul; 25(7):193-8. PMID: 25867038.

20. Yang B, Wang X, Li Z, Qu Q, Qiu Y. Beneficial effects of silver foam dressing on healing of wounds with ulcers and infection control of burn patients. Pak J Med Sci. 2015 Nov-Dec;31(6):1334-9. doi: 10.12669/pjms.316.7734
21. Brown M, Dalziel SR, Herd E, Johnson K, Wong She R, Shepherd M. A randomized controlled study of silver-based burns dressing in a pediatric emergency department. J Burn Care Res 2015 Jul 3. [Epub ahead of print] PMID: 26171678.

22. Ward RS, Hayes-Lundy C, Reddy R, Brockway C, Mills P, Saffle JR. Evaluation of topical therapeutic ultrasound to improve response to physical therapy and lessen scar contracture after burn injury. J Burn Care Rehabil. 1994 Jan-Feb;15(1):74-9. PMID: 8150847.

23. Sar Z, Polat MG, Ozgul B, Aydogdu O, Camcioglu B, Acar AH, Yurdalan SU. A comparison of three different physiotherapy modalities used in the physiotherapy of burns. J Burn Care Rehabil. 2013 Sect-Oct;34(5):e290-6. doi: 10.1097/BCR.0b013e3182789041.

24. Cambier DC, Vanderstraeten GG. Failure of therapeutic ultrasound in healing burn injuries. Burns. 1997 May;23(3):248-9. PMID: 9232286 .

25. Mesquita RL, Silva PIC, Silva SHSM, Oliveira KO, Fontes-Pereira AJ, Freitas JJS, Pereira WCA, Kietzer KS. Effect of low-intensity therapeutic ultrasound on wound healing in rats subjected to thirddegree burns. Acta Cir Bras. 2016 Jan;31(1):36-43. doi: 10.1590/ S0102-865020160010000006.

26. Freitas TP, Freitas LS, Streck EL. Therapeutic ultrasound mechanisms involved in wound healing: a revision. Arq Catarin Med. 2011;40(1):89-93.

27. Weinheimer-Haus EM, Judex S, Ennis WJ, Koh TJ. Low-intensity vibration improves angiogenesis and wound healing in diabetic mice. PloS One. 2014 Mar 11;9(3):e91355. doi: 10.1371/journal. pone.0091355.

28. Demir H, Yaray S, Kirnap M, Yaray K. Comparison of the effects of laser and ultrasound treatments on experimental wound healing in rats. J Rehabil Res Dev. 2004 Sep; 41(5):721-8. doi: 10.1682/ JRRD.2003.08.0131.

29. Rios-Silva M, Trujilo X, Trujilo-Hernández B, Sánchez-Pastor E, Urzúa Z, Mancilla E, Huerta M. Effect of chronic administration of forskolin on glycemia and oxidative stress in rats with and without experimental diabetes. Int J Med Sci. 2014 Mar 11;11(5):448-52. doi:10.7150/ijms.8034.

30. Khorshid FA. Comparative study of keloid formation in human and laboratory animals. Med Sci Monit. 2005 Jul;11(7):BR212-9. PMID: 15990682.

\section{Correspondence:}

Dr. Ruy de Souza Lino Junior

Instituto de Patologia Tropical e Saúde Pública, UFGRua 235, s/n, Setor Leste Universitário

74605-050 Goiás-GO Brasil Tel.: (55 62)3209-6165

Fax: (55 62)3209-6363

ruylino@yahoo.com.br

Received: Mar 21, 2016

Review: May 23, 2016

Accepted: Jun 22, 2016

Conflict of interest: none

Financial source: FAPEG (grant number 2013/0267001091)

${ }^{1}$ Research performed at Tropical Pathology and Public Health Institute, Universidade Federal de Goiás (UFG), Brazil. 